\title{
La verve douce-amère de Lyonel Trouillot
}

\section{Pauline Vachaud}

\section{OpenEdition}

Journals

Édition électronique

URL : https://journals.openedition.org/recherchestravaux/717

DOI : 10.4000/recherchestravaux.717

ISSN : 1969-6434

\section{Éditeur}

UGA Éditions/Université Grenoble Alpes

\section{Édition imprimée}

Date de publication : 15 décembre 2014

Pagination : 135-148

ISBN : 978-2-84310-291-2

ISSN : 0151-1874

\section{Référence électronique}

Pauline Vachaud, «La verve douce-amère de Lyonel Trouillot », Recherches \& Travaux [En ligne], 85 | 2014, mis en ligne le 15 juin 2016, consulté le 29 octobre 2021. URL : http://journals.openedition.org/ recherchestravaux/717; DOI : https://doi.org/10.4000/recherchestravaux.717 


\section{La verve douce-amère de Lyonel Trouillot}

Et ce n'aura pas été une mince affaire que de faire rentrer dans les cases de l'histoire de l'art leurs formes convulsives, polies ou fracassées, qu'il aura d'abord fallu retirer du remous qui les inquiétait, puis débarrasser de l'écume de leur inachèvement, enfin de dégager des concrétions du désespoir qui leur gardait cet air d'éperdu.

Annie Lebrun, Du trop de réalité

«Et le prédicateur, suant à grosses gouttes, enivré par son vin, sa noce, sa verve intarissable. ." Voici, dans tous les romans de Lyonel Trouillot publiés jusqu'alors ${ }^{2}$, la seule apparition explicite du mot "verve». Alors que Colin et Mariéla fuient leur bidonville et le parricide qu'ils viennent de commettre, au petit matin, ils croisent sur leur chemin cet homme de foi dont le discours, ne prêchant d'autres ouailles que les jeunes fuyards puis quelques ouvriers indifférents, sonne "comme une machine tournant à vide» (EH, p. 76). La

I. L. Trouillot, Les Enfants des héros, Arles, Actes Sud, coll. "Générations», 2002, p. 82.

2. Nous n'avons pas été en mesure de nous procurer les textes publiés à Port-au-Prince comme son premier roman Les Fous de Saint-Antoine (1989); notre propos se limitera donc à l'ensemble édité en France, que nous référencerons désormais de la manière suivante : Rue des Pas-Perdus, Arles, Actes Sud, coll. "Générations", 1998 (RPP); Thérèse en mille morceaux, Arles, Actes Sud, "Générations", 2000 (TMM); Les Enfants des héros, ouvr. cité (EH); Bicentenaire, Arles/Montréal, Actes Sud / Leméac, 2004 (B); L'Amour avant que j’oublie, Arles/Montréal, Actes Sud / Leméac, 2007 (AAO); Yanvalou pour Charlie, Arles/Montréal, Actes Sud / Leméac, 2009 $(Y C)$; La Belle Amour humaine, Arles/Montréal, Actes Sud / Leméac, 20II (BAH); Parabole du failli, Arles/Montréal, Actes Sud / Leméac, 2013 (PF). 
«verve», dans un tel contexte, revêt donc un sens religieux doublé d'un caractère radicalement - désespérément - dérisoire.

Si l'on faisait de ce cas notable une généralité, il pourrait alors sembler impropre voire grossier d'appréhender l'œuvre de ce romancier sous l'angle de la verve. Car si le sens du dérisoire n'est pas étranger à la démarche de cet écrivain dont l'inclination tenace à l'utopie n'a d'égale qu'une lucidité sans appel, dans son écriture, rien ne s'apparente à cette chaleur prosélyte, ni aux terreurs du Jugement qui fondent le prêche. Pourtant, dès le prologue du premier roman paru chez Actes Sud, Rue des Pas-Perdus, c'est bien la sensation d'un élan vif, d'un flot de parole emporté qui nous saisit. Où la verve aurait lieu d'être, pour qualifier cette prose vociférante, interpellante, caustique et tendre, révoltée et cynique, émue et lucide. Où l'enthousiasme n'aurait d'autre destinée que de s'en tenir à une impureté douce-amère, au paradoxe de l'aigre-doux.

\section{«Nous sommes les porte-parole de différents nous-mêmes ${ }^{3}$ »}

À traverser l'ensemble de l'œuvre romanesque publiée jusqu'ici par Lyonel Trouillot, un constat s'impose : quel que soit le dispositif énonciatif choisi, tous ses récits sont des «romans à voix ${ }^{4}$ » dans lesquels, face aux aberrations historiques, sociales, économiques, culturelles... d'Haïti, «on enten[d] plus de voix que de causes» ( $B, \mathrm{p}$. Io). Ça parle sans cesse, chez cet auteur, selon des choix énonciatifs où la narration est systématiquement (ou presque) prise en charge par des personnages s'adressant à un ou plusieurs autres, vivants ou morts. Comme si chaque livre constituait l'immersion temporaire dans la relative intimité d'une conversation.

En cela, la verve semblerait tenir de l'oralité du conteur privilégiée par les écrivains de la créolité. Vieille maquerelle, chauffeur de taxi, intellectuel, enfants des bidonvilles, jeune femme naissant à elle-même, avocat carriériste, écrivain, jeune peintre guide touristique, étudiante française en quête d'origine, pigiste... De Rue des Pas-Perdus à Parabole du failli, presque tous les narrateurs mènent un récit qui se veut parole adressée à autrui (ou à l'autre soi-même dans Thérèse en mille morceaux) - pour témoigner, avouer, implorer, justifier, interroger, enseigner, pleurer, tenter de comprendre...

3. L. Trouillot et S. Boutaud de la Combe, Lettres de loin en loin, une correspondance haïtienne, Arles, Actes Sud, 2008, p. 20.

4. «Et si on parlait de ceux qui ne peuvent pas se déplacer» [en ligne], entretien de T. Tervonen avec L. Trouillot, janvier 2002, publié le I/o6/2002. Disponible sur <http://www.africultures. $\mathrm{com} / \mathrm{php} /$ index.php?nav=article\&no=2302> (consulté le oI/o6/2014). 
Dans Rue des Pas-Perdus, c'est la «Nuit de l'Abomination", cette nuit symbolique des violences impensables induites par le régime dictatorial qui pousse trois voix à raconter; dans Les Enfants des héros, le meurtre d'un père violent et la cavale qui a suivi; dans Thérèse en mille morceaux, l'avènement d'une jeune femme à elle-même, à sa liberté propre après des années de conditionnement mortifère; le retour impromptu et tragique des origines reniées dans Yanvalou pour Charlie; le désir d'écrire "vrai», de l'amour et par amour, à la suite d'une rencontre aussi fugitive que cruciale dans L'Amour avant que joublie; la quête d'un récit des origines frotté au réel et à l'idéal d'un présent bouleversant dans La Belle Amour humaine et, pour Parabole du failli, la mort tragique d'un ami's. Ainsi pouvons-nous discerner, à travers cette inclination pour la voix vive, prise dans la ferveur de son adresse, une reconnaissance de dette envers la tradition orale, ce qui, accompagné de l'ancrage dans le réel haïtien, pourrait pousser à envisager la verve de Lyonel Trouillot comme simple signe d'appartenance à la littérature antillaise francophone.

Dès l'anti-Genèse au cœur du prologue de Rue des Pas-Perdus, le propos est nettement marqué par ces traits, hérités de la négritude et perpétués par la créolité, où parole ardente du conteur et forage de la culture propre sont intimement mêlés :

Voilà. Monsieur, cela commença par un grand coup de vent. [...] Oui, cela commença au jour d'avril aux funérailles du président [...]. Oui, tout commença avec ce vent d'avril qui nous fit croire que c'en était fini de la dictature. Les poètes voyaient déjà les chevaux de l'avant-jour courir les grands espaces ouverts par le vent, l'âme hautaine de Bohio ${ }^{6}$ faire pirouette de femme libérée au sommet de Pic Macaya ${ }^{7}$. Camarades, frères, preneurs, sans chamailles ni conciliabules les gens s'embrassaient dans les rues comme en trente-quatre à la fin de la première occupation ${ }^{8}$. [...] Maintenant que les Américains

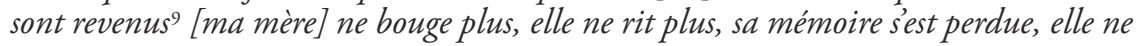

5. Bicentenaire est absent de cette liste car le dispositif énonciatif de ce roman fait exception : ce récit des dernières heures d'un étudiant avant qu'il ne trouve la mort dans une manifestation pacifique contre le pouvoir est mené à la troisième personne. Reste néanmoins que les multiples plongées en discours indirect libre et discours direct libre où le personnage s'adresse à sa mère, à la femme qu'il a aimée etc., rapprochent ce texte du système d'adresse des autres romans.

6. Bohio : un des trois noms que portait l'île d'Haïti avant l'arrivée de Christophe Colomb, repris par Dessalines lors de l'indépendance du pays en 1804, "Haïti-Bohio-Quisqueya».

7. Pic Macaya : montagne haïtienne s'élevant dans le parc national de Macaya situé au cœur de la péninsule de Tiburon.

8. La première occupation des Américains a eu lieu de 1915 à 1934, en réponse à une forte instabilité politique.

9. Il est difficile de dater précisément cette seconde occupation, et la «Nuit de l'Abomination" ne réfère pas, à strictement parler, à un événement historique réel, car elle constitue plutôt la synthèse symbolique des violences politiques en Haïti. Cependant, l'on peut sans doute avancer que la période évoquée est la transition tumultueuse entre la dictature de Duvalier et l'arrivée au pouvoir d'Aristide, dans les années 1990, transition où les Américains eurent un 
parle plus que de ce matin de trente-quatre, elle ne sait pas quils sont revenus ou fait semblant de ne pas savoir quills ont installé des barbelés au bas de la rue des Miracles, que des enfants leur courent après sur la Grande-Rue, sur les dix kilomètres de la route de Delmas, dans les sentiers, que des vieillards leur font des sourires de leurs dents gâtées, que des jeunes filles se font belles pour eux. (RPP, p. 13-15)

Que ce discours préliminaire soit, par ailleurs, énoncé par une voix aussi incarnée qu'anonyme est, sans doute, une autre manière de faire parler le livre au nom du peuple haïtien.

Dans Yanvalou pour Charlie, sur une question moins historique que sociale, la voix de Charlie participe aussi de cette dynamique où se nouent oralité et plongée dans le réel du pays :

Sorry. Ce sont les vieux principes qui m’ont amené vers toi. Avant de me laisser dans la soutane du père Edmond, ma mère me racontait des histoires. Ses histoires, c'était comme des vacances à la campagne, avec un tas de vieux principes. Personne n’aime la campagne, sauf pour les vacances et les vieux principes. Sinon les gens ils y resteraient. Le père Edmond, il critique souvent les «inconscients» qui viennent de la campagne sans savoir ce qu'ils vont trouver ici à Port-au-Prince, et qui construisent des maisons dans la boue, avec de la boue, mangent de la boue, dorment dans la boue, font des enfants dans la boue au milieu des porcs et des poules qui pataugent aussi dans la boue ${ }^{\mathrm{IO}}$. $(Y C, \mathrm{p} .67)$

Comme nombre des personnages de l'auteur, cet orphelin versé dans la petite délinquance par espoir de vivre, un jour, "quelque chose comme une vie normale» (YC, p. 87), incarne le peuple des sans-voix de la société haïtienne. Pour le romancier, «faire entendre ces voix non entendues pour des raisons sociales ${ }^{\mathrm{II}}$ " marque alors la verve de ces discours profus d'une dimension clairement politique, familière de la négritude et de la créolité. Du reste, il n'est pas anodin que cette "petite responsabilité» consistant à donner sa place au «failli» et à "porter la parole ${ }^{\mathrm{r2}}$ » de l'autre soit assimilée par Lyonel Trouillot au fonctionnement du "govi», objet vaudou permettant la communication avec les morts $^{13}$, car ce type de référence rappelle, là encore, l'importance de l'empreinte antillaise. Et quand le locuteur lui-même n'est pas de ceux

rôle important, en soutenant Aristide et en imposant la mise en place d'un plan économique néolibéral.

Io. Contrairement aux autres textes pour lesquels nous nous référerons à l'édition originale, pour ce titre, l'édition de référence sera Actes Sud, coll. «Babel», nº Io69, 20II.

II. Entretien vidéo de P. Beray et C. Marcandier avec L. Trouillot, Paris, le 26/o9/2013, pour Mediapart, publié en ligne le 27/09/2013. Disponible sur <http://www.dailymotion.com/video/ xI58xpo_lyonel-trouillot-parabole-du-failli_news> (consulté le oi/o6/20I4).

I2. Ibid.

13. Voir les développements à ce sujet dans le même entretien de P. Beray et C. Marcandier. Remarquons aussi que ce rituel, sans être explicitement nommé ainsi, est au cœur du mystère d'un des personnages de L'Amour avant que joublie, Raoul, ce dernier ayant accepté d'occuper 
qui subissent la «double peine ${ }^{\mathrm{I}}{ }^{»}$ d'endurer à la fois la misère et le déficit de représentation dans le discours public, comme c'est le cas de Thomas dans La Belle Amour humaine, la manière dont celui-ci dépeint Port-au-Prince à la jeune Anaïse fraîchement débarquée en Haïti constitue encore une forme d'hommage à ces oubliés :

Au centre-ville, le bruit c'est comme la pauvreté, on n'en a jamais fait le tour. La pauvreté, chaque fois qu'on croit la circonscrire dans des quartiers créé pour elle, elle déborde et se lève ailleurs. Le bruit, ici, c'est pareil. Pas moyen de dresser une liste. Les camions-citernes qui râlent et dégoulinent en grimpant les collines. Les grands enfants. Les petits enfants. Les encore enfants qui font des enfants. Les balles perdues. Les fous de Dieu, les annonceurs de fin du monde qui te reprochent de n’avoir pas accepté Jésus pour ton sauveur personnel. [...] Les combats de chiens, les petits d'un côté, les gros de l'autre, comme chez les humains, les petits qui s'enfuient en pleurant leur défaite avant de revenir à la charge pour se faire battre une nouvelle fois par les gros. L'assistance composée de chômeurs et de portefaix qui en ont marre de revoir le même spectacle, même si c'est gratuit, et s'arment de bâtons pour disperser la meute. Et, comme la vie, les bruits ont des humeurs. En prêtant attention, tu pourras distinguer les bruits de la colère de ceux de l'attente et de la fatigue. Ici, les bruits sont la seule preuve de ce dur devoir d'exister et ne chôment jamais. Quand on a perdu tout le reste, reste plus que du temps à perdre. Écoute les bruits du temps perdu. Les chaussures dessemelées qui raclent les pavés. Les cohortes. Les manifs. Les veuves qui défilent au Champ-de-Mars en demandant justice pour des époux assassinés qui ne leur servaient pas à grand-chose de leur vivant mais qu' une mort tragique a rendus sympathiques. ( $B A H$, p. I7-I8)

Ici, la représentation des invisibles ne passe pas par la parole donnée ou rapportée, mais le choix de la fresque reste politique, dans la mesure où il s'agit tout autant de pallier, quoique autrement, le déficit de représentation.

À partir de là, pourtant, on peut toucher une des raisons pour lesquelles l'œuvre de Lyonel Trouillot dépasse le clivage entre littérature française et littérature francophone : elle ne repose pas sur le repli identitaire du "créolement correc $[t]^{15}$ " au nom duquel Césaire, par exemple, fut remisé au statut " "d'anté-créole" » voire "d'anti-créole ${ }^{16} »$. L'un des traits de son univers, qui

une telle fonction non par croyance ni escroquerie, mais par simple générosité envers ceux qui ont à traverser l'épreuve du deuil.

I4. Ibid.

15. A. Lebrun, Pour Aimé Césaire, Paris, Jean-Michel Place, 1994, p. 20, à propos de l'œuvre de A. Césaire telle qu’appréhendée par les écrivains de la créolité comme R. Confiant.

16. «Pensée du divers, pensée de la tolérance, la créolité est en train de faire ses preuves. Et elles sont convaincantes : plus ses sectateurs s'acharnent contre Césaire, plus ils prennent de l'importance dans le monde blanc. [...] Ainsi, d'“anté-créole", à qui l'on reconnaissait encore, il y a quatre ans, d'avoir fait œuvre historique avec la négritude, Aimé Césaire est devenu un anti-créole, à qui la négritude aurait ni plus ni moins servi à éviter de "problématiser son incapacité coloniale d'écrire dans une langue minorée, au-delà du simple constat des interdits 
donne à la verve de son style une autre dimension, c'est, nous y reviendrons, son indépendance obstinée à l'égard de toute idéologie aliénante. Ainsi, comme Césaire, il se veut avant tout chantre de la liberté et, sans nier les spécificités de sa culture, il dépasse amplement les cadres communautaires de la créolité par une prétention à «un universel riche de tous les particuliers ${ }^{17}$ ». Il ne s'agit pas de donner reconnaissance au peuple haïtien parce qu'il est haïtien, antillais ou créole. "Si j'écris, nous dit Lyonel Trouillot lui-même, c'est un peu dans l'idée justement d'une possibilité pour chacun de dire "je" dans un univers qui ne serait ni étouffant ni aliénér ${ }^{18}$." Et, ailleurs, il souligne bien comment, à la manière de Thérèse Décatrel cherchant à "rassembler ses voix ${ }^{19}$ ", sa visée constitue l'articulation du «je» et du «nous», la réalité haïtienne étant plus le substrat de cette dialectique que sa fin :

Sur la thématique, fondamentalement, il y a toujours la tension entre le «je» et le «nous». Le «nous» dans une société comme la société haïtienne est déjà un «nous» impossible, parce que les différences de classes sont telles qu'il n'y a pas de sphère commune de citoyenneté en Haïti. [...] Dans tous les livres que j'écris - surtout en ce qui concerne les romans - c'est justement [mon ambition d'] explorer cette tension entre l'individualité voulant naître à elle-même et l'impossibilité de ce «nous» non existant. [...] Tous mes livres tournent autour de cela et, de préférence en terre haïtienne, parce que la réalité haïtienne est celle que je connais le mieux ${ }^{20}$.

De ce fait, la verve à l'œuvre dans l'ensemble romanesque de cet auteur, et l'oralité qui l'accompagne, touchent à la tentative de rendre vivantes les individualités multiples d'un pays dans cette "sphère commune de citoyenneté" temporaire qu'est le roman, en s'attachant, en premier lieu, aux oubliés; à donner un souffle singulier et puissant à toutes les voix prenant place dans les romans - plus qu'à souscrire à tel ou tel trait typique, et attendu, chez un écrivain des Caraïbes. Ce en quoi il partagerait peut-être, en somme, avec le

langagiers qui régissent la société antillaise”.» (Ibid., p. I8.) Les deux citations convoquées par A. Lebrun sont extraites de L'Éloge de la créolité de R. Confiant.

I7. "Ma conception de l'universel est celle d'un universel riche de tout le particulier, riche de tous les particuliers, approfondissement et coexistence de tous les particuliers.» (A. Césaire, Lettre à Maurice Thorez, citée par A. Lebrun, ibid., p. 33.)

I8. Entretien de G. Tegomo avec L. Trouillot, «La littérature est le lieu de son propre rapport à la liberté» [en ligne], Port-au-Prince, 23 /o6/2009. Disponible sur <http:/www.africultures. $\mathrm{com} / \mathrm{php} /$ index.php?nav=article\&no=8720 $>$ (consulté le or/o6/20I4).

19. Thérèse Décatrel, héroïne de Thérèse en mille morceaux, commente ainsi l'écriture du journal qui l'aidera à sortir libre et heureuse de l'épisode de dissociation qui la tourmente au début du roman : «Ces notes sont à la fois mon repli et mon déploiement, mon ratage et ma mise à jour. À défaut d'une parole droite, j'écris pour rassembler mes voix.» (TMM, p. 17)

20. Entretien vidéo de T.-C. Spear avec L. Trouillot, Port-au-Prince, 23/06/20II, transcrit par M.-D. Grangeois, publié en ligne sur <http://www.lehman.cuny.edu/ile.en.ile/ media/squestions_trouillot.html> (consulté le oI/o6/20I4). 
personnage de l'écrivain de L'Amour avant que j'oublie, le statut «d'écrivain public des voyages intérieurs» $(A A O, \mathrm{p}$. 182). Tout porte à croire que, plus que la défense d'un quant-à-soi créole réducteur, la démarche de Lyonel Trouillot hérite en fait de la position de Césaire, révolté contre l'oppression même - d'où qu'elle vienne :

Si les nègres n'étaient pas un peuple, disons de vaincus, enfin, un peuple malheureux, un peuple humilié, etc., renversez l'Histoire, faites d'eux un peuple de vainqueurs, je crois, quant à moi, qu'il n'y aurait pas de négritude. Je ne me revendiquerais pas du tout de la négritude, cela me paraîtrait insupportable ${ }^{21}$.

À partir de là, il serait plus judicieux de rattacher la verve de ses romans à la mise en jeu de l'altérité (et non de l'antillanité, de la créolité, de l'insularité ou tout autre essentialisme sclérosant ${ }^{22}$ ), altérité qu'il travaille au gré des discours croisés de ses narrateurs-personnages. Peu enclin à défendre l'image ou les valeurs propres, figées d'une identité, il nourrit la vivacité de son style par la traversée littéraire de tout un peuple et de ses voix - ce qui, appliqué à la littérature, rappelle en quelque sorte la «sociologie empirique» (RPP, p. 46) de Gérard, ce personnage discret mais marquant de Rue des Pas-Perdus.

Or, la verve de Lyonel Trouillot ne serait pas si frappante si elle se contentait de reposer sur cette oralité-altérité - de donner voix à ceux qui sont «considérés comme n'ayant rien à dire" ${ }^{23} »$.

\section{«N'oublie pas, mon amour. / Le paradoxe du parfum, c'est qu'il libère ce qu'il capture ${ }^{24}$ »}

C'est aussi parce qu'ils s'appuient sur des valeurs franchement ambivalentes et qu'ils portent en eux un pouvoir d'ébranlement et de libération que l'on peut dire des romans de cet auteur qu'ils ont de la verve. En effet, les postures politiques, idéologiques, morales qu'illustrent ses personnages et ses intrigues

21. Entretien avec Aimé Césaire par Jacqueline Leiner, 1978, cité par A. Lebrun, Pour Aimé Césaire, ouvr. cité, p. 2I.

22. Le point de vue de L. Trouillot sur l'insularité, par exemple, est assez significatif : «La question de l'insularité est pour moi un faux débat, à la limite presque raciste : c'est souvent pour reconnaître une sorte de singularité exotique à l'île et en même temps une impuissance à dire la totalité du monde puisqu'elle ne représente pas cette totalité. C'est un cadeau empoisonné : il y a des relents de colonialisme et un exotisme bon marché dans cette notion d'insularité.» (Entretien de T.-C. Spear cité plus haut.)

23. "Et si on parlait de ceux qui ne peuvent pas se déplacer» (entretien cité).

24. L. Trouillot, Le Doux Parfum des temps à venir, Arles, Actes Sud, coll. «Essences», 20I3, p. 56. 
rendent un ensemble pétri de paradoxes. À l'image des Lettres de loin en loin, cette correspondance mi-réelle mi-fictive avec Sophie Boutaud de la Combe qui synthétise le déchirement entre espoir et lucidité, conviction et autodérision, naïveté, bonté et cynisme, les figures des romans de Lyonel Trouillot incarnent tour à tour - ou en même temps - la foi en l'humanité, en sa beauté, son avenir, sa liberté, mais aussi la désespérance face aux désastres historiques et intimes, le désarroi, la cruauté, la lâcheté... Ainsi, Thérèse en mille morceaux, $L a$ Belle Amour humaine et, dans une certaine mesure, L'Amour avant que joublie cultivent un optimisme tendant vers une "réconciliation rêvée ${ }^{25}$ » où l'espoir de l'auteur en un monde meilleur - aussi désuet que cela puisse paraître pour notre contemporain désenchanté - est très palpable. Pour Thérèse Décatrel, cette femme en «lever d'interdit» (TMM, p. 82), il s'agit de fuir le destin qu'on lui a tracé pour, notamment, ne plus jamais faire «l'amour avec un roi derrière la tête, un évangile, une ville, une histoire, un héritage» et s'autoriser à "habit[er] le léger et l'intense» (TMM, p. Iog). Pour l'écrivain de L'Amour avant que joublie, il s'agit d'accepter d'être réveillé par l'amour, par le trouble qu'il provoque dans sa vie mais aussi dans son écriture :

Moi, je me croyais fidèle à la prudence qui m’a fait fuir l'amitié et le corps des femmes. Je me sens aujourd'hui glisser dans l'infidélité. Tout près de me trahir. Je devrais avoir peur. J'ai, dans les mains, tandis que j'écris, une grande envie de dire : je t'aime. Voudrais-je, parce que toi, revenir au cantique? ( $A A O$, p. 97)

$[\mathrm{Q}]$ ue tu m'introduises dans tous tes quartiers libres. Que tu me bouscules et que je te bouscule./ Vers la vie $(A A O$, p. 137)

Quant à La Belle Amour humaine, c'est l'ouvrage le plus représentatif de cette inclination à l'utopie, presque caricaturale, si n'étaient comme contrepoids, justement, les autres textes plus désabusés. Quand, dès les premières pages, nous apprenons que, dans le village d'Anse-à-Fôleur où Anaïse revient sur les pas de son père et où Thomas, son guide, a "planté ses rêves» $(B A H, \mathrm{p}$. I22), réside un «législateur bénévole et autodidacte» ( $B A H$, p. I4) nommé Justin, qui a travaillé à un "code des nouvelles lois usuelles au service du bonheur", et qu'un des chapitres concerne «l'union libre, le don, la réciprocité et autres vertus quotidiennes» ( $B A H$, p. I4), le tableau est vite campé... Du reste, la fresque à laquelle l'oncle de Thomas a consacré une bonne part de sa vie, «La Belle Amour humaine», synthétise, par la mise en abyme, les principes de ce monde où seuls le bonheur et la beauté de la vie ont lieu d'être - l'homme

25. "Il y a des livres dans lesquels il y a une vision plus optimiste, où il semble y avoir cette réconciliation rêvée, et d'autres qui sont peut-être plus pessimistes ou plus réalistes, dans lesquels on voit l'échec de cette tentative de créer un "je" qui soit dans une harmonie avec la collectivité, avec le "nous".» (Entretien de T. C. Spear déjà cité.) 
d'affaires sans scrupules Robert Montès et le colonel tortionnaire Pierre André Pierre, qui avaient cru pouvoir prendre place dans ce village ayant été, purement et simplement, effacés du paysage. Un monde où le législateur est dit un «Socrate» n'imposant pas ses lois (BAH, p. 49), proposant que soit offert aux vieillards sur le point de mourir un "cadeau de départ" fait de rire et d'amour charnel (BAH, p. 55); un monde où «le devoir [est] de merveille» $(B A H$, p. I69) et où les seules questions qui vaillent sont : «Ai-je fait un bel usage de ma présence au monde?» $(B A H, \mathrm{p} .24)$, "à quel chant donner sa part de voix?» (BAH, p. 167), «De quoi parlions-nous?» (BAH, p. Io6); où les armes tombent en échange d'une nuit d'amour $(B A H, \mathrm{p}$. I23), où l'on pratique "l'art de la route et de la rencontre» $(B A H$, p. I24) et où se nourrit «l'envie de naître au hasard des chemins» (BAH, p. I47). Quoi de plus pastel, de plus bleu comme l'amour bleu de la chanson du même nom ouvrant L'Amour avant que joublie? Voilà, peut-être, un exemple abouti de l' "anarcho-lyrisme" (RPP, p. 46) juste évoqué dans Rue des Pas-Perdus ${ }^{26}$ et tragiquement incarné par Pedro, cet "artiste maudit» (PF, p. 23) de Parabole du Failli, "Quichotte et baladin, innocent jusqu'à la bêtise» $(P F, \mathrm{p} .75)$. Cet idéal de vivre ensemble libertaire, noué par un sens poétique quotidien et partagé, a de quoi faire pâlir toutes les tentatives de réenchantement actuelles - et relève, pour qui se réclame de la "pensée célibataire ${ }^{27}$ " du temps, d'une certaine obsolescence idéologique et littéraire pouvant prêter à sourire. Mais si l'on compte que ce livre fonctionne en contrepoint de la désolation sans appel qui ressort de Rue des Pas-Perdus, Bicentenaire, Les Enfants des héros et, dans une certaine mesure, Yanvalou pour Charlie; si l'on reconnaît par ailleurs une certaine légitimité littéraire et éthique au «sabotage passionne ${ }^{28}$ » et donc, à la force subversive et créatrice du désir et de l'amour, alors, peut-être, pouvons-nous reconnaître à cet "anarcho-lyrisme» une portée moins anecdotique, moins pittoresque

26. À propos de Gérard, déjà cité plus haut, ce professeur de mathématiques qui s'est retiré de l'enseignement parce que la réalité «refusait de s'adapter aux théorèmes» (RPP, p. 88)

27. A. Lebrun désigne par cette formule la «[p]ensée de l'efficacité qui n’a d'yeux que pour elle-même, pensée de la manipulation qui prétend se préserver de tout ce qui n'est pas elle, pensée de la stratégie qui n'a d'autre fin que d'imposer sa solitude pour prévenir tout embrasement passionnel.» (Du trop de réalité [2000], Paris, Gallimard, coll. "Folio essais», 2005, p. 296. Dans le même passage, elle fait ainsi le lien entre une "désérotisation généralisée» (où l'éros est entendu au sens le plus fort du désir), la servitude volontaire et le moralisme.

28. «Pour s'opposer à ce bonheur dans la soumission en train de s'imposer en art de vivre, ne restent que les rares êtres qui, d'instinct, lui échappent. [...] Y aurait-il même des mots, des idées, des lieux que cette pensée célibataire n'a pas réussi à neutraliser complètement, il dépend de chacun d'aller les lui reprendre sauvagement. Telle est la nouvelle "reprise individuelle" qui attend ceux qui se souviennent des Travailleurs de la nuit. Si quelque chose peut encore commencer - ce qui n'est pas forcément probable - c'est uniquement par ce sabotage passionnel.» (A. Lebrun, ibid., p. 297.) 
qu'il n'y parait. Et ce d'autant plus que, comme nous venons de le souligner, les contre-exemples ne manquent pas. Parce que l'étudiant pacifiste, dans Bicentenaire, tombe sous les doubles assauts de l'armée et des chimères - ces jeunes mercenaires des quartiers pauvres parmi lesquels compte son propre frère -, tout le livre semble pouvoir être résumé par deux phrases :

Et voilà qu'il doit se contenter d'une ville sale et ensanglantée dans laquelle personne ne connaît plus personne. $(B$, p. 97)

[Les chimères] savent que le triomphe inévitable de la colère du juste est un mythe épuisé, un piège pour les naïfs. ( $B, \mathrm{p}$. Iı)

De même, Colin, des Enfants des héros, incarne un cynisme amer : "Une chose que j'ai apprise, personne ne peut servir d'exemple. Personne n'a tort et personne n'a raison. C'est la vie qui a tort.» (EH, p. I23) Quant à la mort du jeune Charlie, elle participe d'une ironie tragique qui rappelle, en mode mineur, les extrémités de Rue des Pas-Perdus et qui finit de neutraliser, comme en complément de Bicentenaire, toute velléité à changer le monde. En effet, c'est parce que l'un de ses compères d'orphelinat, Nathanaël, cerveau de leur bande de gentils cambrioleurs, a rallié un groupe de révolutionnaires idéalistes et armés que, par un concours de circonstances malheureuses, Charlie meurt sous les balles d'un de ces militants - un enfant des beaux quartiers ayant embrassé le gauchisme "pour ne pas ressembler à son père» ( $Y C, \mathrm{p} .30)$, dont la révolte a été "apprise dans les livres» ( $Y C$, p. I22) mais qui, face à la réalité, se laisse submerger par la peur. Finalement, alors que le groupe d'orphelins cherchait "les moyens de s'acheter une étoile" (YC, p. 84) et que l'un d'eux a souhaité qu' existe "une étoile pour chaque vivant» $(Y C$, p. 88$)$, nulle brèche n'a réussi à poindre dans l'impasse du paysage de ces enfants de la misère : «Entre une misère qui dure toujours et une gloire qui dure pas longtemps et qui finit dans le bain de sang, y a pas vraiment d'alternative.» (YC, p. 87)

Dès lors, si l'histoire de Yanvalou pour Charlie constitue comme la réénonciation du thème de Rue des Pas-Perdus, c'est que, dans ce premier roman centré sur les affrontements terribles entre tenants du pouvoir et opposition, c'est à l'échelle de toute une ville - et de tout un pays - que la lueur de l'espoir semble résolument éteinte. Pour la vieille maquerelle ( $\mathrm{I}^{\mathrm{re}}$ voix) que l'on découvre au moment où elle a renoncé à toute activité et vit dans l'obsession des malheurs dont elle a été le témoin plus ou moins passif, le nihilisme a vaincu :

Les seules règles à ne point violer sont la misère et la violence. Ne vous fâchez pas, messieurs-dames, mais voilà notre permanence. [...] Il n'y a plus d'ici. Il n'y a plus qu'un grand cimetière où des morts dansent le zouk et le compas direct. [...] Mais oui, mes beaux messieurs-dames, ce n'est rien qu'un grand cimetière où des morts jouent à tuer les morts, à vider leurs cercueils de leurs ossements. (RPP, p. I39-I40) 
De même, si le chauffeur de taxi ( $2^{\mathrm{e}}$ voix $)$ a survécu à la "Nuit de l'Extermination", c'est au prix d'une de ses jambes, perdue dans le cloaque qui lui a servi d'abri. Depuis, il est devenu l'infirme, instrumentalisé et volé par ses petits-enfants. Et, comme la vieille maquerelle, il reste à jamais atteint, hanté, par les souvenirs de cette nuit.

Enfin, pour la $3^{\mathrm{e}}$ voix, celle de l'intellectuel employé des postes, les stigmates peuvent paraitre moins profonds, puisque durant la nuit en question, il n'est témoin des exactions commises que par les échos des comptes rendus réguliers de son ami Gérard, chez qui il est refugié. Il reçoit même, avec le renversement du pouvoir, une proposition de promotion. Mais, durant cette nuit, il a pu expérimenter combien les soubresauts de l'Histoire vouaient son amour, son désir, sa liberté à l'asphyxie :

Qu'est-ce que vivre quand la mort met la vie en demeure? Quand on n'a pas les moyens de ses désirs. Quand des voix extérieures qui ne sont pas des voix, rien que des ordres opposés, des tyrannies de fossoyeurs, choisissent nos rencontres, coordonnent nos parcours. André dirait que cela n'a point d'importance, que l'histoire n'a pas le temps de s'arrêter à ces détails. Mais nulle histoire n'est une petite histoire. (RPP, p. I37)

Parce que cette "Nuit de l'Abomination» condense une histoire de vengeance contre vengeance, de tortures contre tortures, sous couvert de justice et de légitimité populaire, elle symbolise combien les idéologies, jouets des rages inassouvies des hommes (et vice-versa), sont aussi mortifères qu'impuissantes à rendre les jours meilleurs. De là, si l'«anarcho-lyrisme» a encore lieu d'être dans ces textes où le tragique pur prend le dessus, c'est sans doute dans son versant critique. La longue litanie de la maquerelle vitupérant «au nom du pain» les tenants du pouvoir, quels qu'ils soient, ainsi que leurs suiveurs, en constitue un bel exemple :

[C]e qui reste, ce qui n'a pas bougé, pire que le pion ou les morpions, pire que tout, pire que vous-mêmes, mes messieurs-dames parce que c'est elle qui vous nourrit et qui vous crée tels que vous êtes, c'est la misère. Alors permettez que je crache sur les drapeaux et les parades, sur vos titres et sur vos slogans. Au nom du pain. Sur vos haines et sur vos mensonges. Au nom du pain. Sur les rats que vous devenez quand il vous prend de mordre et de souffler dans la misère de qui n'a rien à mordre et pas de souffle pour souffler. Sur vos diatribes, vos têtes d'affiche, sur les galons que vous inventez parce qu'à force de mentir on finit par croire en la vérité du mensonge. [...] Permettez que je crache sur vos monuments. Au nom du pain. Vous n'êtes pas foutus de leur faire des fontaines qui coulent. Tant il est vrai, mes messieurs-dames, que vous n'avez à leur offrir que des orgies d'apocalypse, des jérémiades de poitrinaires. Et vos gueules de couteaux de pharmacie qui viennent trancher dans leur misère. Et eux comme des chiens errants qui n'ont plus de place pour errer parce que la misère ça prend toute la place [...]. (RPP, p. I27-I28) 
Entre cette rage radicale et l'innocente générosité de La Belle Amour humaine, la synthèse peut être difficile à envisager. Pourtant, c'est parce qu'il assume la contradiction et, surtout, la complexité des êtres, des phénomènes, des événements, que Lyonel Trouillot maintient malgré tout un univers consistant : un monde qui, en définitive, opte pour la nuance, la subtilité de l'aigre-doux - à l'instar de ce "parfum» des "doux temps à venir ${ }^{29}$ " qui, en libérant ce qu'il capture, symbolise le nœud de paradoxe propre à toute intention, tout idéal, tout geste. Néanmoins, que la tonalité globale de son œuvre romanesque soit douce-amère ne signifie en rien que l'enjeu soit nul, neutre ou anodin. Malgré cette complexité assumée (ou, sans doute plutôt, grâce à elle), chaque texte reconduit un désir d'ébranlement, ce en quoi toute l'œuvre peut être placée sous le signe du mouvement, du déplacement - synonymes, pour Lyonel Trouillot, de libération ${ }^{30}$.

Nous touchons là l'une des dernières raisons pour lesquelles nous pouvons convoquer la verve, pour qualifier le travail romanesque de cet auteur. À porter une attention particulière à son style, on remarque qu'y règne un transport qui vise à déplacer, toujours ailleurs et autrement, les coordonnées des êtres et de leur monde - à relancer, sans cesse, la matière même de la libération qu'est le mouvement. Ainsi, «l'énormité poétique ${ }^{31}$ » qui traverse la plupart de ses textes mériterait à elle seule un article - voire davantage. Même si certains titres représentent plus significativement cette propension à l'excès, où la langue s'emballe, se meut en une déferlante inassouvissable (comme Rue des Pas-Perdus et Bicentenaire), il demeure que cette tendance à laisser la parole jaillir sans autre frein que son propre épuisement parvient à se manifester dans chaque roman. Pour ne prendre qu'un exemple, on peut s'arrêter sur cette page de Bicentenaire où la verve tient notamment à une nette réminiscence du Cahier d'un retour au pays natal de Césaire ${ }^{32}$. Il s'agit d'un long commentaire sur les banderoles qui habillent les toits de Port-au-Prince :

Mille textes croisés, promesses et ratures, menaces et merveilles : Jésus est là... le président a dit... ne ratez pas l'ouverture... Tous les langages du temps qui passe et ne passe pas, de la ville à jamais agonisante, ayant tout raté, jusquà la gestion des ordures et du commerce des morts, la ville balbutiante et mal débarbouillée, gargouillant dans ses tréfonds de miasmes, de coups tordus, de faux pas; tatouée

29. L. Trouillot, Le Doux Parfum des temps à venir, ouvr. cité.

30. Pour s'en convaincre, on se reportera notamment à cette phrase de Bicentenaire, où est évoqué le sens de la marche pour les manifestants : "Chaque pas qu’ils font donne la preuve de l'existence du mouvement ; chaque pas qu'ils font conteste la fixité du malheur. » (p. 69)

3I. Pour reprendre, en l'adaptant plus ou moins à notre propos, une notion employée par A. Lebrun dans Du trop de réalité, ouvr. cité, p. I07-I09.

32. Plus particulièrement, de l'ouverture du poème. 
dans son ciel de faux bons du Trésor, comme le corps du petit, dressant inventaire de son déficit de paroles vives, donnant lecture de ses minables acquisitions et promesses d'acquisition, puisant dans son compte de verbiages pour habiller son ciel de mots [...]. La ville trébuchante, parlant par écrit, par pattes de mouche, hors sujet. La ville contresens, ouverte comme une plaie, étalant sans vergogne ses fautes de syntaxe, ne disant rien qui vaille, mais refusant d'être muette : Jésus est là... le président a dit... ne ratez pas l'ouverture... La ville à court de propositions, ruminante, bégayante [...]. [I]l n'est point d'or caché dans le ventre des mots. Les mots sont creux. Les morts ne parlent plus. Les mots n'ont nulle présence et n'attendent pas de réponse. Bicentenaire, soldes, colloque pourrissent sur les banderoles comme un corps de pendu, un cadavre desséché qui refuse de tomber. (B, p. 68)

Le vertige des accumulations ne prend fin qu'au moment où le discours se replie sur la conscience de la vanité du langage - de celui des banderoles, mais aussi, en définitive, du langage en soi; et si ce dégonflement de l'emphase, cette exténuation finale, laisse le dernier mot à l'amertume, il demeure que la ferveur qui anime cette éruption discursive persiste dans l'oreille, sans attendre longtemps avant de jaillir de nouveau. Dans le cas présent, le retour de la vague n'a lieu qu'une petite page plus loin.

Pour finir, on pourrait aussi évoquer le tremblement du sens qui fait aussi force d'ébranlement, mais il ne relève pas précisément de la verve, car il a moins trait à l'emportement, à l'énergie et à l'adhésion qu'implique celle-ci que, plus modestement, à une mise en suspens, au vacillement de l'indécidable, qui arrête pour interroger. Ainsi en est-il de la folie - ou non - du chauffeur de taxi dans Rue des Pas-Perdus; de la responsabilité - ou non - des enfants parricides dans Les Enfants des héros; des causes incertaines de la mort de l'homme d'affaires Robert Montès et du colonel Pierre André Pierre dans La Belle Amour humaine. Mais il s'agit là d'un autre champ d'étude qu'il n'y a pas lieu d'ouvrir plus avant.

Ainsi, en faisant du roman le lieu de l'effusion de voix éminemment singulières quoique manifestes du réel haïtien; en dégageant un parfum d'ensemble qui mêle l'amertume la plus désabusée et la tendresse la plus rare - où l'impureté de nos conditions, de nos désirs, de nos actes et idéaux, est courageusement embrassée; en fondant le style sur une éthique de l'excès et du mouvement qui engage une soif d'ébranlement et de libération inextinguible, les romans de Lyonel Trouillot incarnent une verve qui, pour ardente qu' elle soit, ne verse ni dans le caprice ni dans le pur brio car elle assume pleinement l'imperfection humaine qui est la sienne et que, loin d'être un écran posé devant l'Histoire et l'intime, elle constitue au contraire un étai solide pour affronter le réel du monde, des autres et de soi. 
Ce n'est alors pas le moindre des paradoxes que d'avoir recours à la verve pour "écouter le silence de l'autre ${ }^{33}$ " mais, puisque la «mise en bouche est toujours de l'ordre de la trahison ${ }^{34} »$, trahison pour trahison, paradoxe pour paradoxe, autant que cet effort de relais passe par une fougue, une sauvagerie même, qui sauve non seulement de la pauvreté mais aussi de la normalisation.

33. Entretien de P. Beray et C. Marcandier cité plus haut.

34. Ibid. 